\title{
RPS3A positively regulates the mitochondrial function of human periaortic adipose tissue and is associated with coronary artery diseases
}

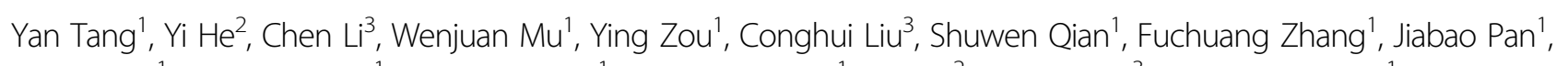
Yina Wang ${ }^{1}$, Haiyan Huang ${ }^{1}$, Dongning Pan ${ }^{1}$, Pengyuan Yang ${ }^{1}$, Ju Mei ${ }^{2}$, Rong Zeng ${ }^{3}$ and Qi-qun Tang ${ }^{1}$

\begin{abstract}
Pericardial adipose tissue, which comprises both epicardial adipose tissue (EAT) and paracardial adipose tissue (PAT), has recently been recognized as a novel factor in the pathophysiology of cardiovascular diseases, especially coronary artery disease (CAD). The goal of this study was to evaluate differences in the brown-like characteristic and proteome among human EAT, PAT, and subcutaneous adipose tissue (SAT) to identify candidate molecules causing CAD.

Uncoupling protein 1 (UCP-1) and other brown-related proteins were highly expressed in pericardial adipose tissue but was weakly expressed in SAT from the same non-CAD patient. Moreover, pericardial adipose tissues displayed a higher thermogenesis than SAT. However, brown-related genes were lower in CAD pericardial fat. Remarkably, there were lower levels of metabolic enzymes involved in glycolysis, tricarboxylic acid cycle, and fatty acid metabolism in pericardial adipose tissues of CAD. EAT is an organ adjacent to aortic root without anatomy barriers, which differs from PAT. We found that the expression of ribosomal protein S3A (RPS3A) was decreased in human EAT as well as in mouse perivascular adipose tissue (PVAT). Knockdown of RPS3A significantly inhibited adipocyte differentiation in preadipocytes and impaired the function of mitochondria in mature adipocytes. Moreover, RPS3A knockdown in mouse periaortic adipose tissue impaired browning of PVAT, accelerated vascular inflammation, and atherosclerosis progression. Mechanistically, RPS3A can migrate to the mitochondria to maintain the function of brown adipocytes. These findings provide compelling evidence that RPS3A was a key factor for modulating the brown fat-specific gene UCP-1 and carbon metabolic enzymes in EAT for preventing CAD.
\end{abstract}

\section{Introduction}

Obesity is one of the main causes of metabolic syndrome, and is associated with chronic inflammation and

\footnotetext{
Correspondence: Ju Mei (meiju@xinhuamed.com.cn) or Rong Zeng (zr@sibs.ac.cn) or Qi-qun Tang (qqtang@shmu.edu.cn)

${ }^{1}$ Key Laboratory of Metabolism and Molecular Medicine of Chinese Ministry of Education, Department of Biochemistry and Molecular Biology of School of Basic Medical Sciences and Department of Endocrinology of Zhongshan Hospital, Fudan University, 200032 Shanghai, China

${ }^{2}$ Department of Cardiothoracic Surgery, Xinhua Hospital, Shanghai Jiaotong University of Medicine College, 200032 Shanghai, China

Full list of author information is available at the end of the article.

These authors contributed equally: Yan Tang, Yi He, Chen Li.
}

cardiovascular disease ${ }^{1}$. However, according to the Framingham Heart Study, metabolic risk factors are more associated with omental adipose tissue than with subcutaneous adipose tissue $(\mathrm{SAT})^{2}$. These differences are plausibly due to differences in adipose tissue distribution and metabolism.

There are two major types of adipose tissue: white adipose tissue (WAT) and brown adipose tissue (BAT). WAT is composed of adipocytes with a large, single fat droplet and is presumed to be the main depot for lipid storage, whereas BAT contains several smaller fat droplets and numerous mitochondria and is involved in heat

\section{(c) The Author(s) 2018}

(c) (i) Open Access This article is licensed under a Creative Commons Attribution 4.0 International License, which permits use, sharing, adaptation, distribution and reproduction cc) in any medium or format, as long as you give appropriate credit to the original author(s) and the source, provide a link to the Creative Commons license, and indicate if changes were made. The images or other third party material in this article are included in the article's Creative Commons license, unless indicated otherwise in a credit line to the material. If material is not included in the article's Creative Commons license and your intended use is not permitted by statutory regulation or exceeds the permitted use, you will need to obtain permission directly from the copyright holder. To view a copy of this license, visit http://creativecommons.org/licenses/by/4.0/. 
production $^{3}$. BAT is present throughout life in rodents, whereas in humans, it was thought to rapidly involute postnatally, essentially disappeared within the first years after birth ${ }^{4}$. However, positron emission tomography (PET) and X-ray computed tomography (CT) showed that active uncoupling protein 1 (UCP1)-expressing adipocytes, including brown adipocytes or beige cells, are localized close to the clavicular, periaortic, cervical, and suprarenal regions in adulthood ${ }^{5,6}$. Both brown and beige adipocytes are functionally thermogenic and are considered to be promising new therapeutic avenue to combat atherosclerosis and obesity.

In addition to the classical BAT existing in the interscapular, recent studies have found that perivascular adipose tissue (PVAT) is a vasoactive organ with functional characteristics similar to BAT, and plays an important protective role in the pathogenesis of atherosclerosis $^{7}$. PVAT directly abuts the adventitia of blood vessels and actively communicates with the vascular wall to regulate vascular function and inflammation ${ }^{8}$. Loss of PVAT surrounding the vasculature causes temperature loss and endothelial dysfunction, and promotes atherosclerosis in mice ${ }^{7}$. Other studies have found that cold exposure and beta- 3 adrenergic receptor ( $\beta 3$-AR)-mediated BAT activation enhances the selective uptake of fatty acids from triglyceride (TG)-rich lipoproteins into BAT, subsequently accelerating the hepatic clearance of the cholesterol-enriched remnants and protecting from atherosclerosis 9 .

In humans, epicardial adipose tissue (EAT) predominantly functions as PVAT for the coronary arteries, and displays high rates of both lipogenesis and lipolysis. It is thought to serve as a local fat storage depot, storing excess free fatty acids as TG at times of excess and releasing them to the heart for substrate utilization in times of metabolic stress ${ }^{10}$. In addition, paracardial adipose tissue (PAT) is the fat surrounding the parietal pericardium, termed "mediastinal fat" or "thoracic fat". Recent data in humans suggest that EAT and PAT may be "beige" in morphology, with features of both WAT and BAT $^{11-13}$. Measurements of EAT and PAT volume have suggested that the thickness of pericardial adipose tissue, which comprises both EAT and PAT, may serve as a risk factor and biomarker to predict the early stages of atherosclerosis and coronary heart disease $(\mathrm{CAD})^{14-16}$. Given the different depot-specific gene expression profiles in "brown" adipocytes, the purpose of this study was to provide a better understanding of the putative thermogenic function of human EAT and PAT using tissues obtained from patients undergoing open heart surgery with and without CAD.

Locally produced adipokines by pericardial adipose tissue might reflect or affect cardiovascular pathology due to its proximity to coronary arteries ${ }^{14}$. We hypothesized that there are differences in the proteome patterns in pericardial adipose tissue from patients with and without CAD. Among the proteins, ribosomal protein S3A (RPS3A) exhibited 3-fold lower expression in EAT from CAD patients. RPS3A, a component of the ribosomal small subunit (40S), shows high sequence homology in mammalian cells and is localized in both the nucleus and cytoplasm $^{17}$. RPS3A also has multiple biological functions unrelated with the ribosome. Previously known as v-fos transformation effector gene, RPS3A is an inducing factor for the oncogenic cellular transformation of Rat-1 fibroblasts by $\mathrm{v}$-fos ${ }^{18}$. In addition, RPS3A is highly expressed in most tumors ${ }^{19,20}$. However, the mechanism underlying the role of RPS3Acin thermogenesis remains unknown.

In this study, we found that in non-CAD patients, UCP1 was relatively abundant in EAT and PAT, both depots possess smaller lipid size characteristic of those found in vitro in beige lineage adipocytes. The browning and thermogenesis of EAT and PAT were significantly decreased in patients with CAD. The decreased expression of RPS3A in EAT was associated with a "brown to white" change in mature adipocytes and vascular dysfunction. These results may shed light on the role of pericardial adipose tissue in the physiopathology of atherosclerosis and CAD.

\section{Results}

Human pericardial adipose tissue displays some characteristics of BAT compared with the subcutaneous depot

As shown in Supplementary Table S1, a total of 58 CAD patients were the case group, and 64 non-CAD patients undergoing valvular heart surgery without $C A D$ were the control group. The CAD and control groups were comparable among several covariates including age, gender, body mass index (BMI), waist size, smoking status, and metabolic syndrome. Individuals with CAD were older than those without CAD, and women had a lower incidence of developing CAD. In addition, waist size was significantly higher, whereas BMI was similar between CAD patients and the control group. CAD patients were more likely to have a history of hypertension. However, history of diabetes mellitus, serum TG, total cholesterol (TC), high-density lipoprotein (HDL), and low-density lipoprotein (LDL) were not significantly different between CAD patients and controls (all $p>0.05$ ), which may be explained by the older population. From these characteristics and laboratory findings, we hypothesized that other factors may modulate atherosclerosis besides classical factors.

EAT, PAT, and SAT were obtained from the same subjects undergoing heart surgery without CAD. Hematoxylin and eosin (H\&E) staining and immunohistochemistry (IHC) showed that the tissue morphologies 


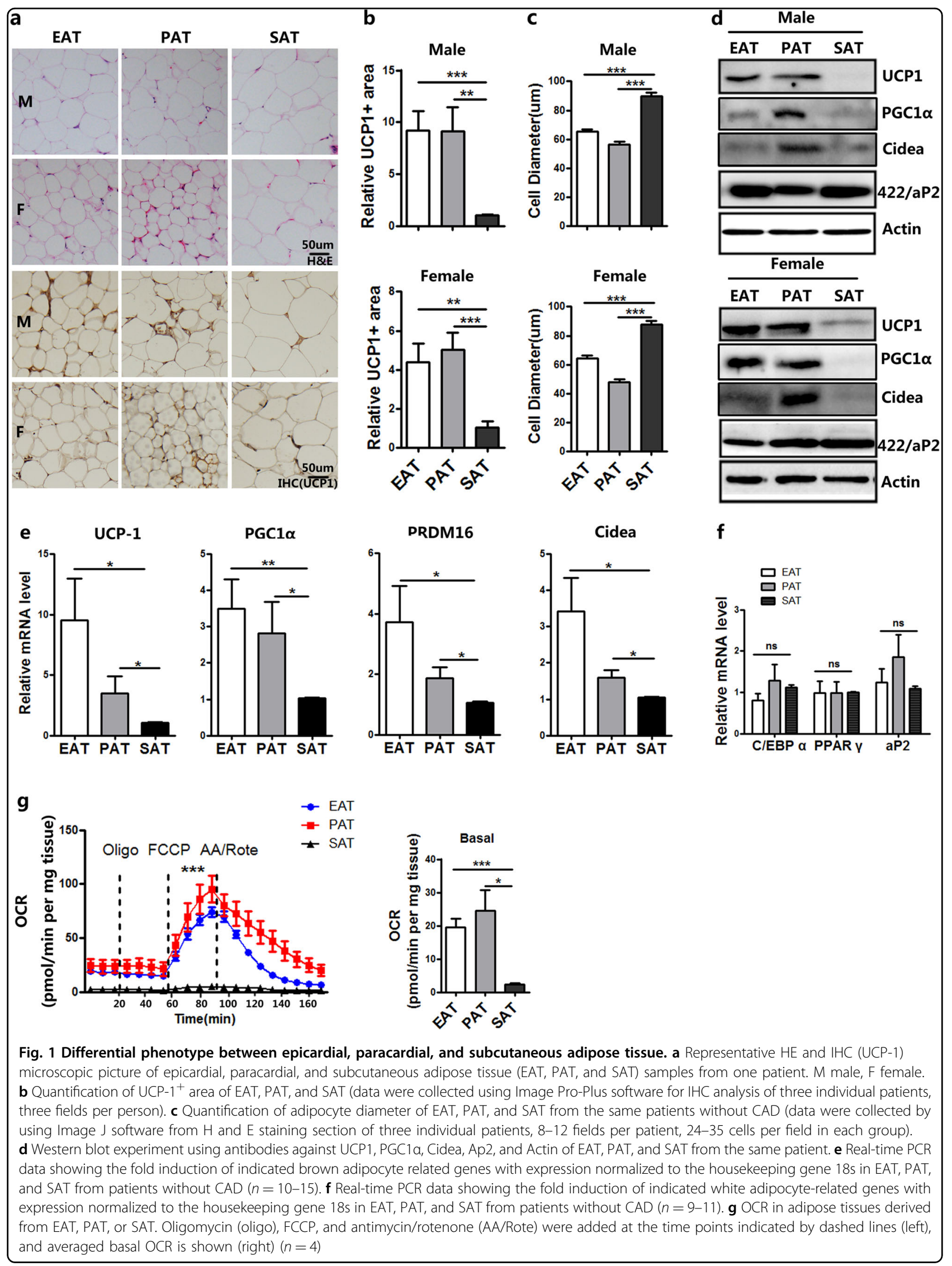


were generally similar in the three depots (Fig. 1a). However, the cross-sectional area of adipocytes in the EAT and PAT were significantly less than that in SAT (Fig. 1c). Multilocular lipid droplets were observed in some adipocytes in PAT from female patients, whereas only unilocular lipid droplets were observed in SAT adipocytes from the same patients (Fig. 1a and Supplementary Fig. S1a). Regions of EAT and PAT were stained positively for UCP1, but weak or no staining for UCP1 was observed in SAT (Fig. 1a, b). The protein expressions of UCP1, PGC1 $\alpha$ and Cidea were measured by western blotting and found to be relatively high in pericardial adipose tissue, whereas the expression level of the WAT marker aP2 was the same (Fig. 1d). qPCR analysis revealed a significantly higher expression of UCP1, PGC1 $\alpha$, PRDM16, and Cidea in the pericardial adipose tissue compared to the subcutaneous depot, but a similar expression of C/EBP $\alpha$, PPARy, aP2 (Fig. 1e, f). We also found that the oxygen consumption rate (OCR) of pericardial adipose tissues were significantly higher than SAT (Fig. 1g).

To further verify the brown-like characteristics of pericardial adipose tissue, stromal vascular fraction (SVF) was isolated from adipose tissues and differentiated into mature adipocytes in vitro. Primary SVF derived from EAT, PAT, and SAT was cultured and differentiated toward beige adipocytes. SVF from PAT and SAT showed higher adipogenic ability compared with EAT (Supplementary Fig. S2a). Other studies have shown that beige adipocytes have smaller lipid droplet; we evaluated morphological changes using Oil Red O staining. Adipocytes differentiated from PAT-SVF demonstrated the reduced size of lipid droplets, concomitantly the increased protein level of UCP1 and the mRNA levels of UCP1, PGC1 $\alpha$, and PRDM16 (Supplementary Fig. S2b-e). These results provided strong evidence confirming the brown-like characteristics of pericardial adipose tissue.

\section{Metabolic disorders in EAT and PAT from CAD patients}

Having identified the brown-like characteristic of pericardial adipose tissue, we reasoned that the browning of pericardial adipocyte may be decreased in CAD patients. Indeed, UCP1 staining of pericardial adipocytes from CAD patients showed much less brown staining than non-CAD patients (Fig. 2a, b). Changes were further confirmed by the decreased mRNA levels of UCP1 and PGC1 $\alpha$ (Fig. 2e, f) and diminished UCP1, PGC1 $\alpha$, and Cidea protein expressions in CAD patients (Fig. 2c and d). However, the expressions of UCP1 and PGC1 $\alpha$ were the same in non-pathological epicardial adipose tissue (EAT$\mathrm{NC}$ ) from CAD and non-CAD patients (Supplementary Fig. S3a). All CAD patients had SYNTAX score II by applying the Cox proportional hazards model. These CAD patients were divided into three groups and the mRNA levels of UCP1 and PGC1 $\alpha$ were detected. Patients with the highest SYNTAX score had a lower expression of UCP1 in both EAT and PAT (Fig. 2g, h). These data indicate a "brown-to-white" shift of pericardial adipose tissue in CAD patients.

To describe the systemic regulations and find useful biomarkers of EAT and PAT in CAD patients, the EAT or PAT proteome with or without CAD were analyzed by tandem liquid chromatography-tandem mass spectrometry (LC-MS/MS) using a label-free strategy. From 10 pairs patients, there were a total of 52 proteins from EAT, and 85 proteins from PAT that were differentially expressed between CAD and non-CAD patients (Fig. 3a). Comparison of the two data sets indicated that there were eight proteins overlapped in both EAT and PAT, which was confirmed by qPCR analysis (Fig. 3b). In order to give a proteomic snapshot, we outlined the changed proteome and discovered that enzymes participating in intermediate metabolism were preferentially changed in EAT and/or PAT of CAD (Fig. 3c), which agrees with that of the previous researches ${ }^{21}$. More interestingly, most of the changed proteins were concentrated in the mitochondria (Fig. 3d), illustrating the mitochondrial oxidative stress play an important role in $\mathrm{CAD}^{22,23}$. Indeed, most of the enzymes involved in glycolysis, the tricarboxylic acid (TCA) cycle, and fatty acid metabolism showed a decreased trend in CAD patients (Fig. 3e). These results uncover a previously unrecognized and potentially extensive role of pericardial adipocyte metabolism in regulation of atherosclerosis.

\section{RPS3A is required for brown adipogenesis to maintain epicardial adipocyte dynamics}

To determine the mechanism underlying the "brown to white" changes of pericardial adipose tissue, we next identified the potential candidate proteins in EAT, which is derived from aortic root. In the clinic, the presence of aortic atherosclerosis can be used as an additional marker for predicting $\mathrm{CAD}^{24}$. In our study, we also found calcified plaque around the aortic root in CAD compared with non-CAD patients (Supplementary Fig. S3b). First, we examined the expression of adrenergic receptor beta $(\mathrm{AR} \beta)$, thyroid hormone receptor beta (ThR $\beta)$, M1 macrophage-related gene (TNF $)$, and M2 macrophagerelated gene (Mrc1) in EAT from non-CAD and CAD patients. However, there were no significant changes with exception of the inflammation-related gene TNF $\alpha$ (Supplementary Fig. S3c). Second, based on our proteomic data, the abnormal regulation of the metabolic pathway was shown in CAD tissues (Fig. 4a). The five proteins, Col1a1, RPS3A, HSD17B12, Crip1, and LCN2, which were changed specially in EAT (Fig. 4a, Supplementary Fig. S3d). Given the multifunction of ribosome protein, we determined its functional role during brown adipocyte 


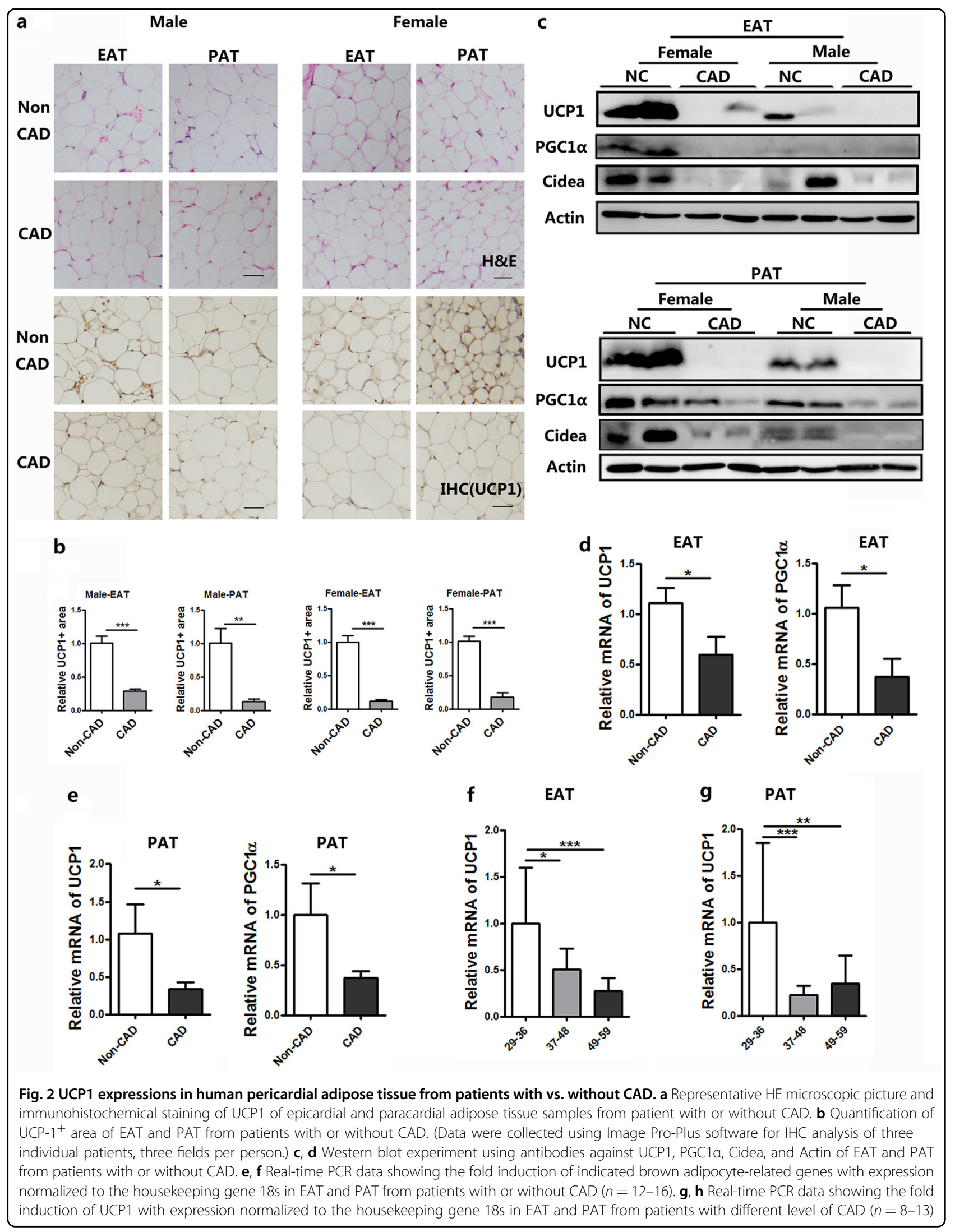




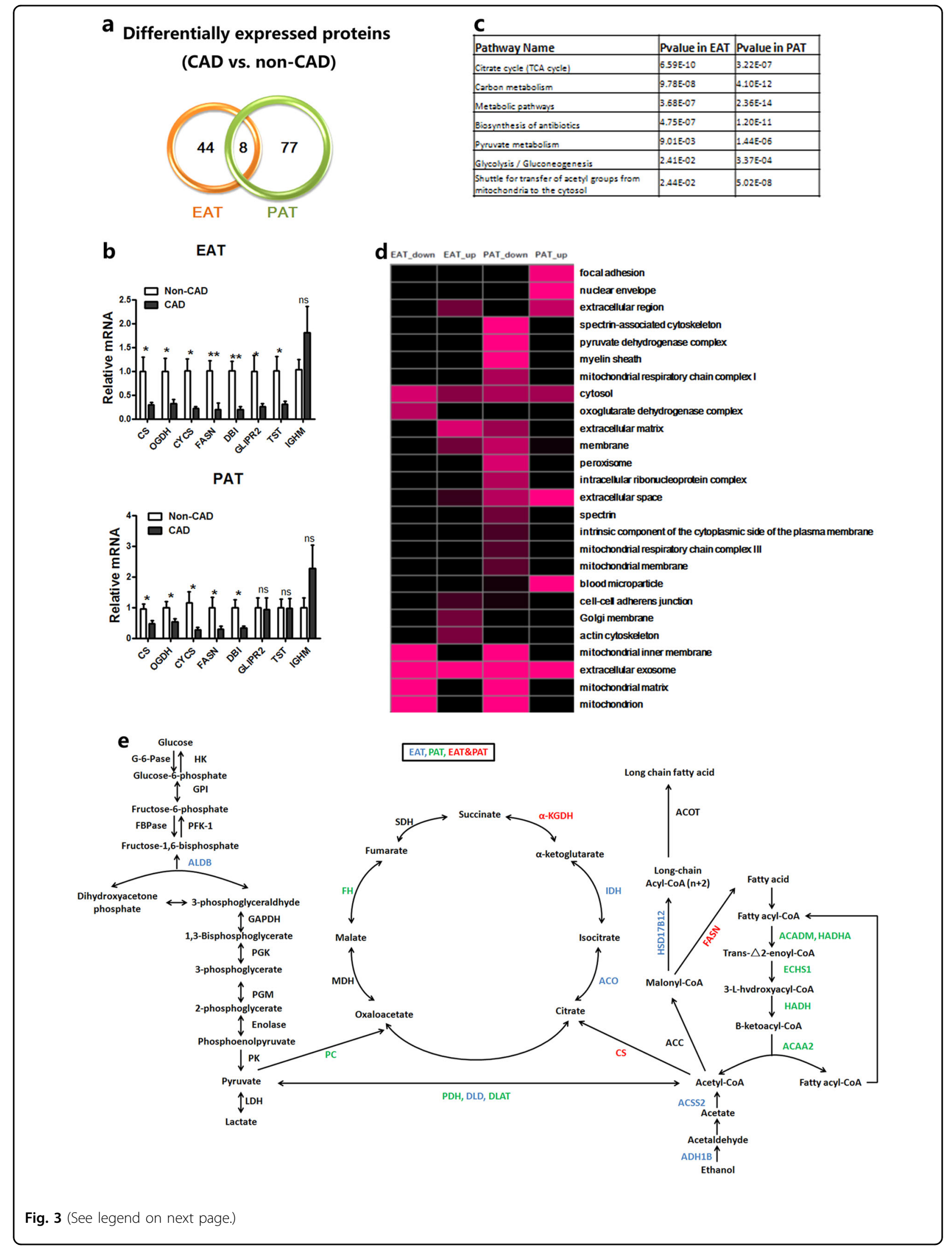


(see figure on previous page)

Fig. 3 Heat map showing the expression of the literature-curated mitochondrial function genes in the epicardial and pericardial adipose tissue of patients with vs. without CAD in the RYGB profiling experiments. a Venn map of the differentially expressed proteins found in EAT or PAT tissues comparing CAD and non-CAD cases. $\mathbf{b}$ Real-time PCR validation of genes selected from the proteomic analysis $(n=8-12)$. $\mathbf{c}$ Enriched metabolic-associated KEGG pathways with downregulated expressed proteins in EAT or PAT tissues were shown by table. $\mathbf{d}$ Enriched GO-CC terms (hypergeometric test, $p$ value $<0.05$ ) in down- or upregulated expressed proteins of EAT or PAT between CAD and non-CAD cases. Gradations of purple stand for the significant degrees (-log ( $P$ value, 10)) of enrichment. e Proteins identified changed in CAD samples by proteomic survey are marked in blue (only EAT), green (only PAT), and red (EAT and PAT)

differentiation. Real-time PCR and western blotting analysis confirmed that RPS3A was significantly decreased in CAD tissue (Fig. 4b, c).

$\mathrm{ApoE}^{-1-}$ mice have been widely used as an atherosclerosis animal model. Thus, we next investigated RPS3A expression level in PVAT of WT fed ND (normal diet), WT fed HFD (high-fat diet), $\mathrm{ApoE}^{-1-}$ fed ND, and $\mathrm{ApoE}^{-1-}$ mice fed HFD for 4 months. We found that HFD feeding induced WT mice browning process in PVAT, which was associated with an increased expression of RPS3A. Importantly, $\mathrm{ApoE}^{-1-}$ atherosclerosis mice had decreased expression of UCP1, PGC1 $\alpha$, PRDM16, Cidea, and RPS3A (Fig. 4d-f). Confocal microscopic analysis was conducted to demonstrate the tissue location of RPS3A. Colocalization between RPS3A and mitochondria was detected, while colocalization between RPS3A and tubulin was not exist (Fig. 4g).

To further explore the function of RPS3A in the process of adipogenesis, specific Stealth ${ }^{\mathrm{TM}}$ RNAi of RPS3A was used to knockdown its expression in immortalized brown preadipocytes (Fig. 5a). Knocking-down RPS3A inhibited the adipogenic ability and decreased the expression of adiocyte-specific markers (Fig. 5b, c). Further investigations showed a decreased expression of RPS3A in 3T3-L1 and C3H10T1/2 cell line both inhibited adipogenesis (Supplementary Fig. S4a-f). Previous findings indicate that the preadipocyte will first undergo mitotic clonal expansion (MCE) and subsequently terminal differentiation during the adipogenesis, while $C / E B P \beta$ is required for both of these events ${ }^{25}$. The RNAi effect of RPS3A on DNA synthesis was evaluated by using EdU labeling. As shown in Fig. 5d, e, RPS3A knockdown did not cause less EdU incorporation. Adipogenesis is controlled by a transcriptional cascade composed of a large number of transcriptional factors, including CCAAT/enhancer-binding proteins (C/EBPs), peroxiaome proliferator-activated receptor $\gamma$ (PPAR $\gamma)$, signal transducers and activators of transcription (STATs), and Kruppel-like factor (KLF) proteins ${ }^{26}$. C/EBP $\beta$ is induced very early in adipocyte differentiation. Then it activates the expression of C/EBP $\alpha$ and PPARy, two critical pro-adipogenic transcription factors, by binding to their promoters ${ }^{27}$. Previous studies have shown that knockdown of C/EBP $\beta$ and PPAR $\gamma$ prevent adipocyte differentiation ${ }^{28,29}$. We found that downregulation of RPS3A reduced the expression of $\mathrm{C} / \mathrm{EBP} \beta$, and subsequent PPAR $\gamma$ and $422 / \mathrm{aP} 2$ proteins (Fig. 5f). Collectively, these results indicated that RPS3A may play a dominant role in preadipocyte differentiation.

\section{RPS3A promotes the function of mature brown adipocytes to inhibit atherosclerosis}

Knockdown of RPS3A in preadipocytes inhibited adipogenesis, so as to suppress more stem cell differentiation into mature adipocytes in the physiological or pathological state, which is consistent with clinical studies that HIV-related lipodystrophy syndrome is associated with an increased risk for the development of cardiovascular disease $^{30}$. On the other hand, clinical studies have found that a larger pericardial fat volume is associated with a higher risk of CAD. We hypothesized that RPS3A might be a potential regulator for maintaining the function of brown adipocytes. To test this presumption, we examined the effects of RPS3A knockdown or overexpression on the brown adipocytes by performing RNA interference for RPS3A (SiRPS3A) or infecting adipocytes with Ad-RPS3A in mature adipocytes. As expected, RPS3A mRNA and protein expression were significantly reduced by SiRPS3A (Fig. 6a, c). Oil Red O staining showed that knockdown of RPS3A increased lipid droplet size (Fig. 6b). In addition, the protein expression of UCP1 and PGC1 $\alpha$ was significantly reduced by siRPS3A (Fig. 6c). Furthermore, knockdown of RPS3A decreased OCR in brown adipocytes (Fig. 6d). In contrast, overexpression of RPS3A by Ad-RPS3A increased small multiple-lipid droplet size (Fig. 6e) and effectively rescued the UCP1 expression and OCR level of brown adipocyte caused by white adipocyte induction cocktail without indometacin and T3 (Fig. 6f, g).

To gain deeper insights into the importance of RPS3A in PVAT to vascular function, we next injected adenovirus LacZ shRNA or RPS3A shRNA in the PVAT around the aortic arch (Fig. 7a). Knockdown of RPS3A decreased the activation of PVAT as evidenced by reduced UCP1 protein content per area and reduced thermogenic genes (Fig. 7b, c). In addition, we also depleted RPS3A in inguinal adipose tissue of WT mice by infecting AdshRPS3A into the left SAT, while Ad-shLacZ was injected into the right SAT of the same mice. Injection of RPS3A 
a

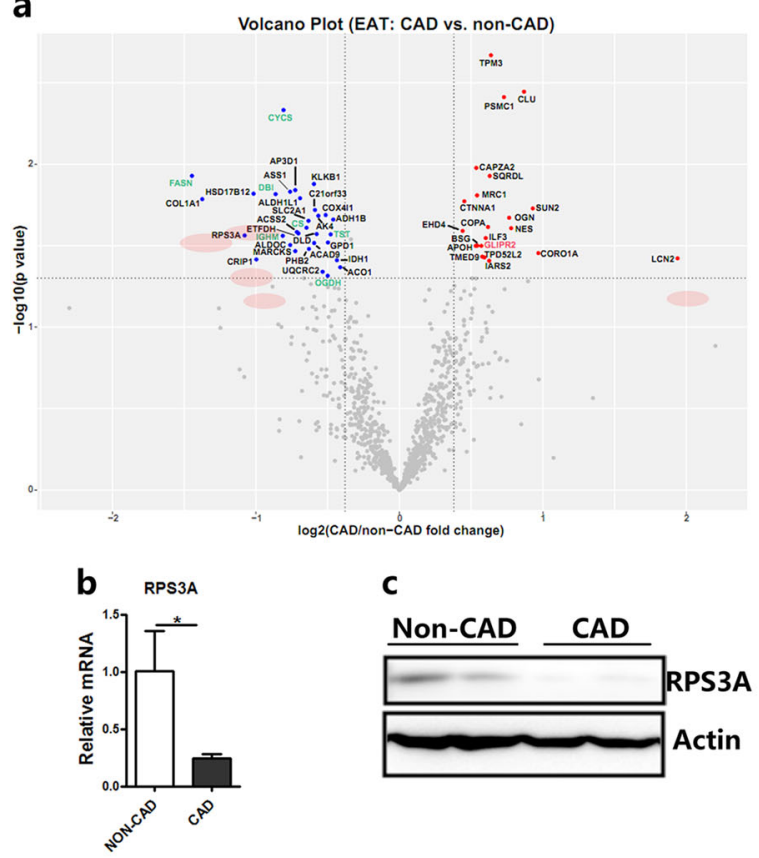

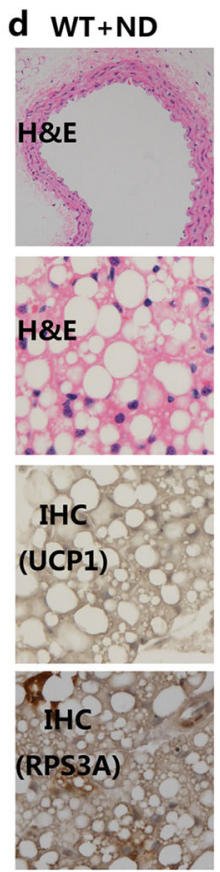
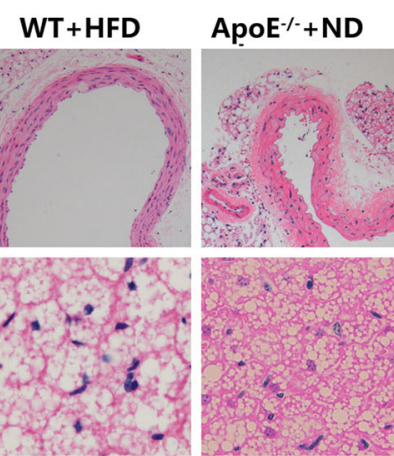

ApoE ${ }^{-/}+$HFD
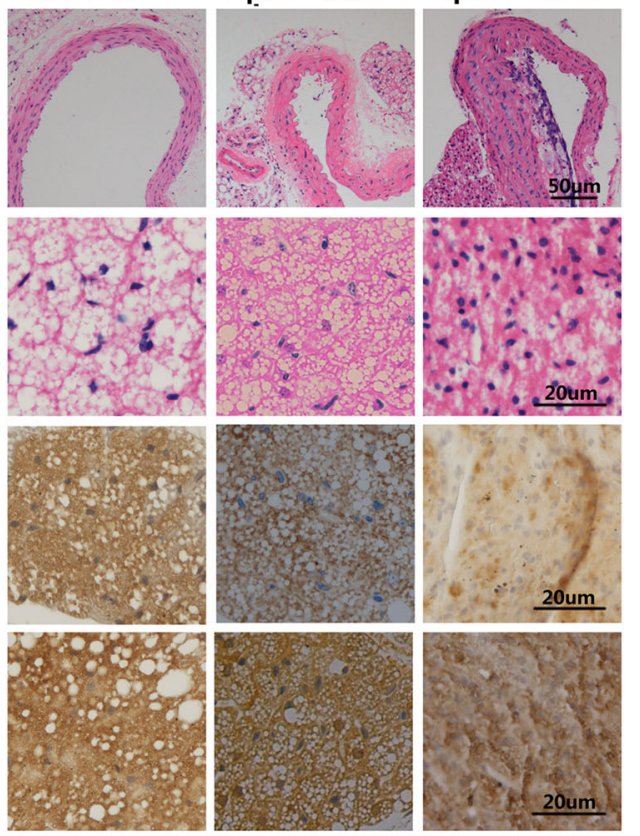

e
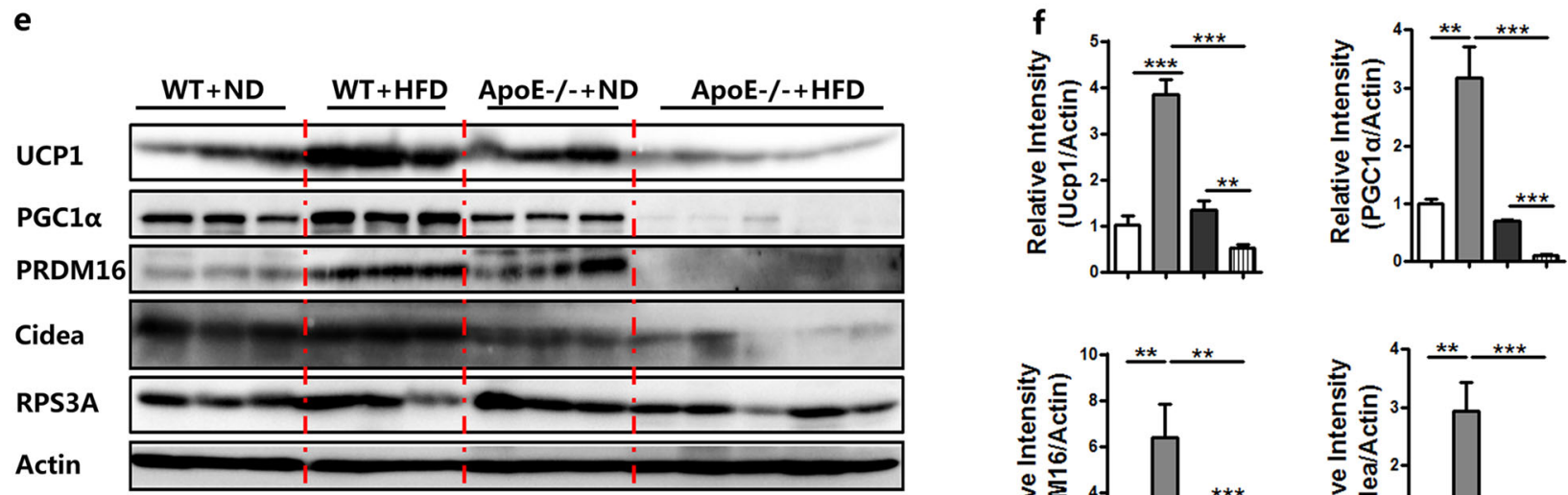

g

RPS3A

DAPI
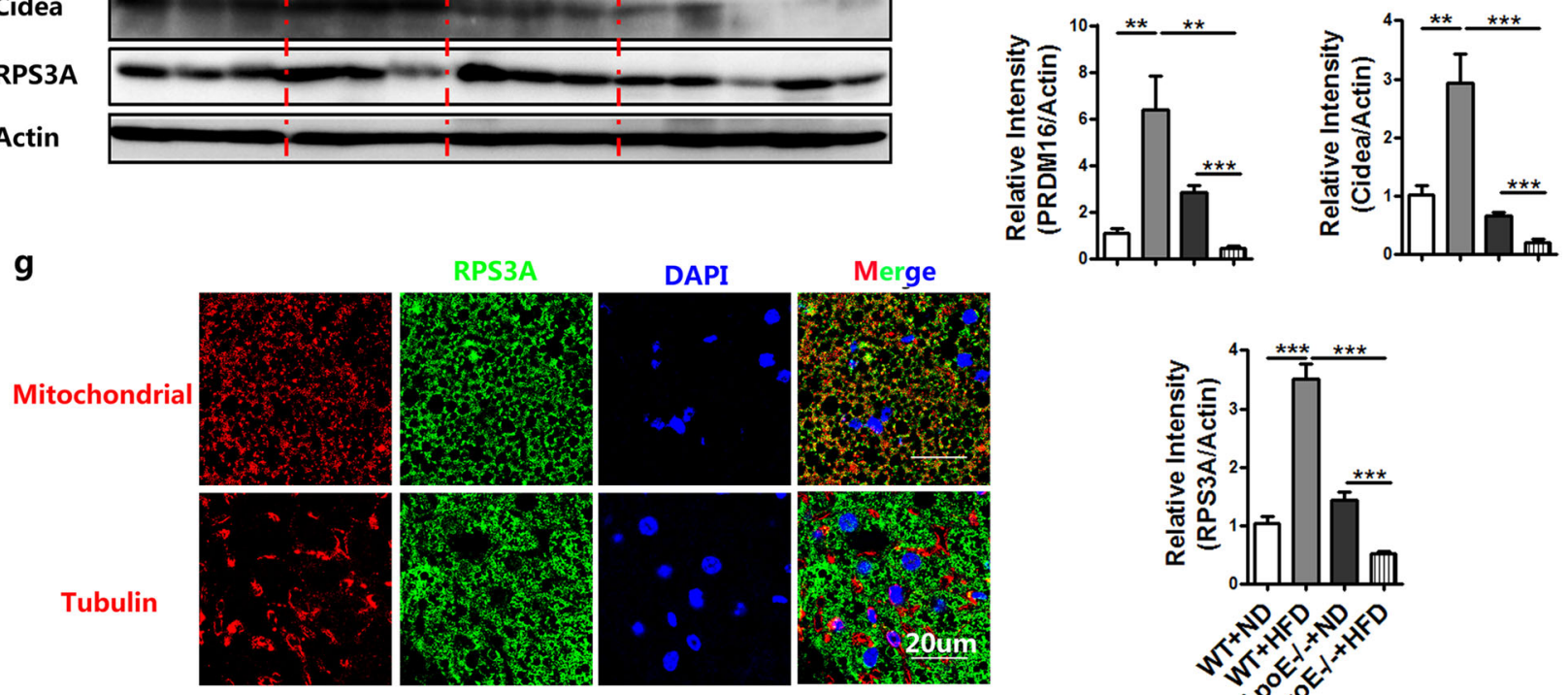

Merge
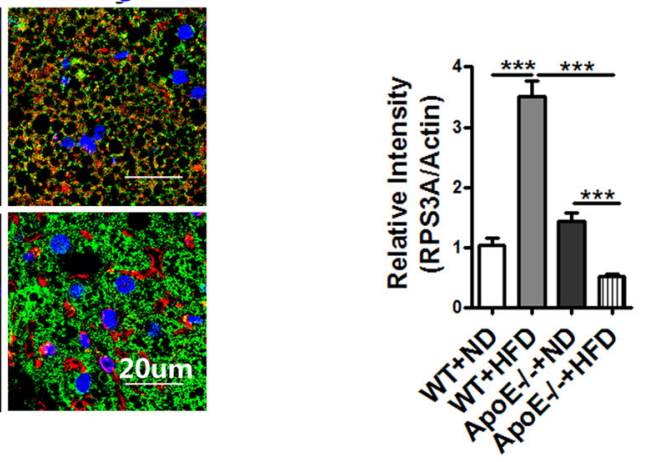

Fig. 4 (See legend on next page.)

shRNA into SAT resulted in larger size of adipocytes from H\&E staining of adipose tissues (Supplementary Fig. S5a). UCP1 protein content per area of inguinal adipose tissue was decresed injected with RPS3A shRNA, suggesting the inactivation of beige adipose tissue (Supplementary Fig. S5a). Moreover, knockdown of RPS3A caused a 
(see figure on previous page)

Fig. 4 The expression of RPS3A is decreased in perivascular adipose tissue to inhibit stem cell adipogenesis. a Volcano plot of EAT proteome comparing CAD and non-CAD patients. The gene symbols of 31 downregulated proteins and 21 upregulated proteins in CAD cases were marked. b Real-time PCR validation of RPS3A from the EAT proteomic analysis of patients with or without CAD $(n=7-9)$. c Western blot experiment using antibodies against RPS3A and Actin of EAT from patients with or without CAD. $\mathbf{d}$ H\&E staining and Immunohistochemical staining of UCP1, RPS3A of vascular (top), and adipose tissue (middle and bottom) sections obtained from 8-week-old WT littermate control mice fed normal chow diet (WT +

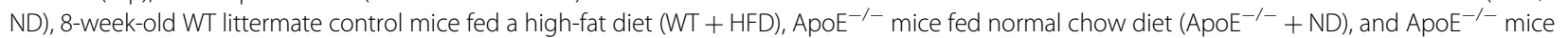
fed a high-fat diet (ApoE ${ }^{-\prime-}+$ HFD) for 4 months. e Western blot experiment using antibodies against UCP1, PGC1a, PRDM16, Cidea, RPS3A, and Actin of PVAT from mice as indicated above. $\mathbf{f}$ Relative gray intensity of the band (E) was quantitated using Image J software $(n=6)$. $\mathbf{g}$ Confocal microscopic images of PVAT sections immunostained for mitochondria/tubulin (red), RPS3A (green) and DAPI (blue)

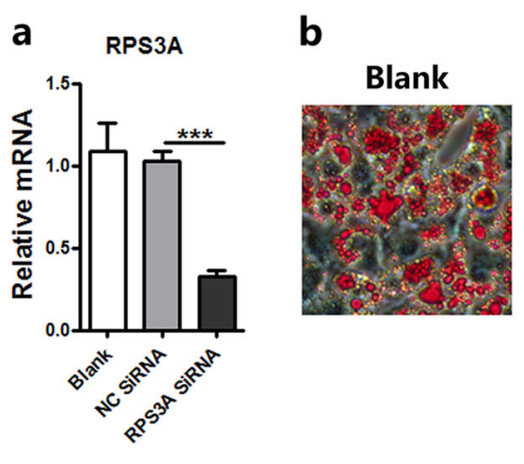

d

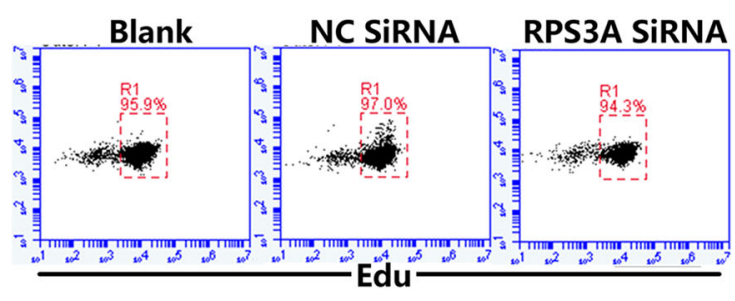

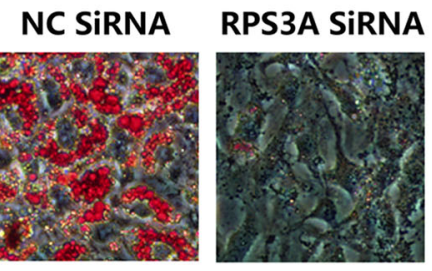

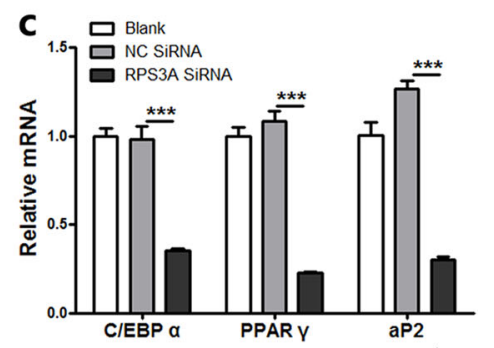

e
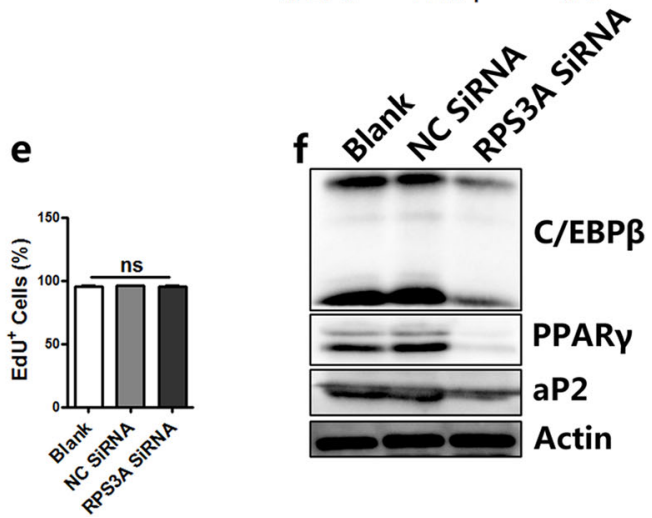

Fig. 5 RPS3A knockdown in brown preadipocyte suppresses adipogenesis. a Brown preadipocytes were treated with RNAi before adipogenic induction and collected for qPCR to determine relative mRNA expression levels of RPS3A $(n=3)$. $\mathbf{b}$ Oil Red O staining of adipocytes differentiated from brown preadipocytes on day 8. c qPCR to determine the decreased mRNA expression of adipocyte markers $(n=3)$. $\mathbf{d}$ and $\mathbf{e}$ Flow cytometric analysis of EdU+ cells in brown preadipocytes $(n=3)$. $\mathbf{f}$ Brown preadipocytes were treated with RNAi before adipogenic induction and Western blot experiment using antibodies against C/EBPß, PPARY, aP2, and Actin on day 4

decreased expression of UCP-1, PGC1 $\alpha$, PRDM16, and Cidea (Supplementary Fig. S5b). All these results indicated that RPS3A knockdown decreased the browning process in SAT during cold exposure. In line with the results obtained from human samples, inhibition of RPS3A in the PVAT increased the thickness of the vasculature and contributed to vascular inflammation by increasing the levels of TNF $\alpha$, interleukin 1 beta (IL-1 $\beta$ ), IL-6, interleukin adhesion molecule 1 (ICAM1), and vascular cell adhesion molecular 1 (VCAM1) (Fig. 7d, e). BAT could secrete factors that act locally and systemically to influence fuel and energy metabolism ${ }^{31}$. Adiponectin secreted by adipocytes could act on the blood vessels to inhibit neointima formation and macrophage inflammation, and suppressing the hepatic expression of the transcription factor SREBP2, thereby leading to reduced cholesterol synthesis and attenuation of hypercholesterolemia $^{32}$. Interestingly, treatment of aortic vascular with adipocyte-derived conditioned medium (CM) pretreated with adenovirus RPS3A significantly decreased the mRNA expression levels of TNF $\alpha$, IL-1 $\beta$, IL-6, ICAM1, and VCAM1 (Fig. 7f, g), and demonstrated that overexpression of RPS3A in brown adipocytes inhibited inflammation of vascular, thereby protecting from atherosclerosis.

\section{RPS3A migrates to the mitochondria to maintain the function of mitochondria in brown adipocytes}

BAT dissipates energy in the form of heat, which relies on a high abundance of mitochondria as well as high 


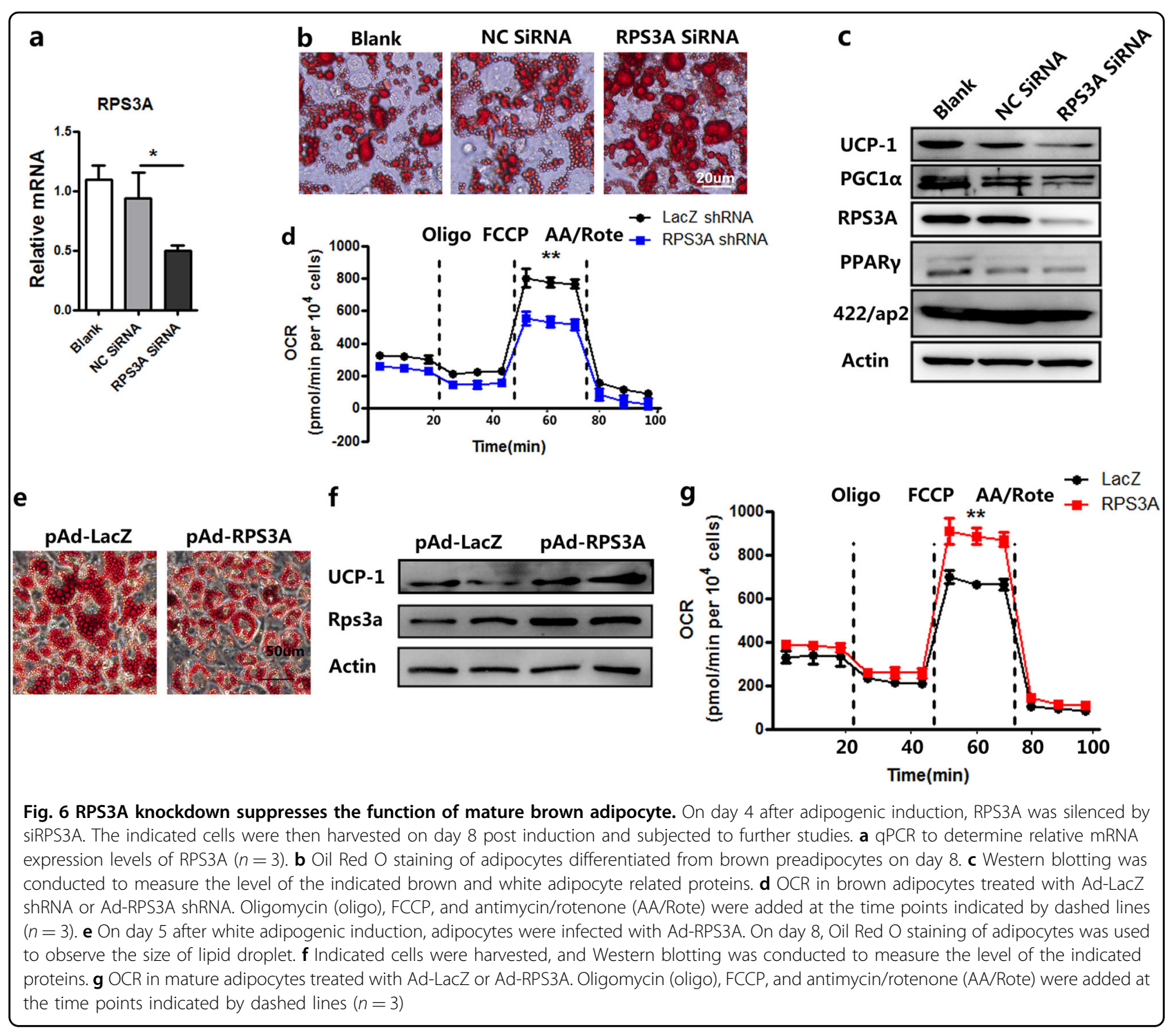

levels of electron transport chain complexes and UCP1 within the mitochondria. Brown preadipocytes were induced and then developed into mature adipocytes with NC SiRNA or RPS3ASiRNA. Knockdown of RPS3A decreased mitochondria biosynthesis as shown by mitochondrial immunofluorescence (IF) staining (Fig. 8a, b). To further verify the effect of RPS3A in mitochondria, we detected the expression of cytochrome $\mathrm{C}$ in mature adipocytes, and found that RPS3A knockdown decreased the protein level of cytochrome C, whereas RPS3A overexpression increased its expression (Fig. 8c, d). Next, we investigated the subcellular localization of RPS3A protein by confocal IF analysis using RPS3A and mitochondria antibodies. Interestingly, RPS3A and mitochondria protein expression overlapped in the mature brown adipocytes, whereas RPS3A protein accumulated in the cytosol of the pre-adipocytes (Fig. 8e). To future confirm the mitochondrial translocation of RPS3A, we performed a cytosol/mitochondria fractionation assay. As shown in Fig. 6f, the mitochondrial translocation of RPS3A distinctly increased in the process of brown adipogenesis compared with unstimulated cells.

\section{Discussion}

BAT is essential for adaptive thermogenesis and energy expenditure in human infants and then disappears in adults $^{33,34}$. However, there is accumulating evidence demonstrating that BAT, which is present in areas close to the clavicular, periaortic, cervical, and suprarenal regions, is activated in response to cold exposure ${ }^{4,6}$. In addition, recent studies have shown that PVAT, similar to $\mathrm{BAT}$, is a heat-generating organ that is critical for the maintenance of intravascular temperature and vascular homeostasis $^{7}$. Activation of BAT in hypertriglyceridemic 


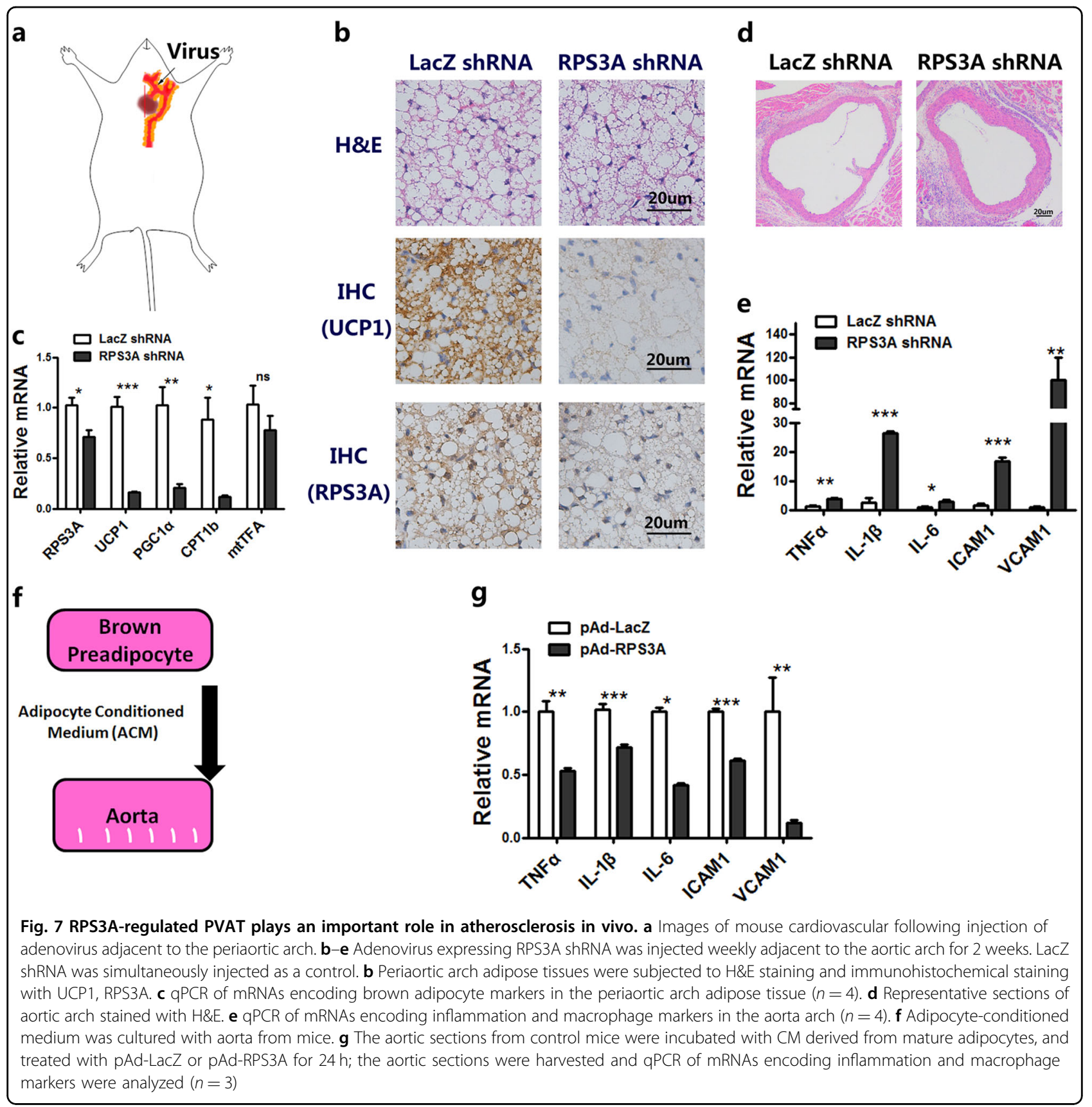

mice by cold strongly reduces plasma TG, illustrating the importance of BAT in lipoprotein metabolism, which is a major risk factor for atherosclerosis ${ }^{35}$. BAT itself is responsible for the uptake of TG-derived fatty acids, leading to the formation of cholesterol-enriched lipoprotein remnants, which are subsequently cleared from the plasma by the liver via binding the ApoE to the hepatic LDL receptor ${ }^{36}$. In humans, daily cold exposure for 20 min for 90 days reduces TC and LDL-cholesterol in hypercholesterolemic individuals ${ }^{37}$. However, there is no such study to compare the browning characteristics between the pericardial adipose tissue and SAT from the same patient without CAD. We are the first to report that pericardial adipose tissue of EAT and PAT exhibits brown cell features while simultaneously expressing high levels of some genes typifying brown adipocytes. Previous work in rodents indicated that BAT is greater in females than males $^{38}$. In our study, we demonstrated that multilocular lipid droplets exist in $50 \%$ of female PAT from histology analysis (Supplementary Fig. S1). Using western blot analysis, we present evidence that UCP1 expression was higher in pericardial adipose tissue from females than 


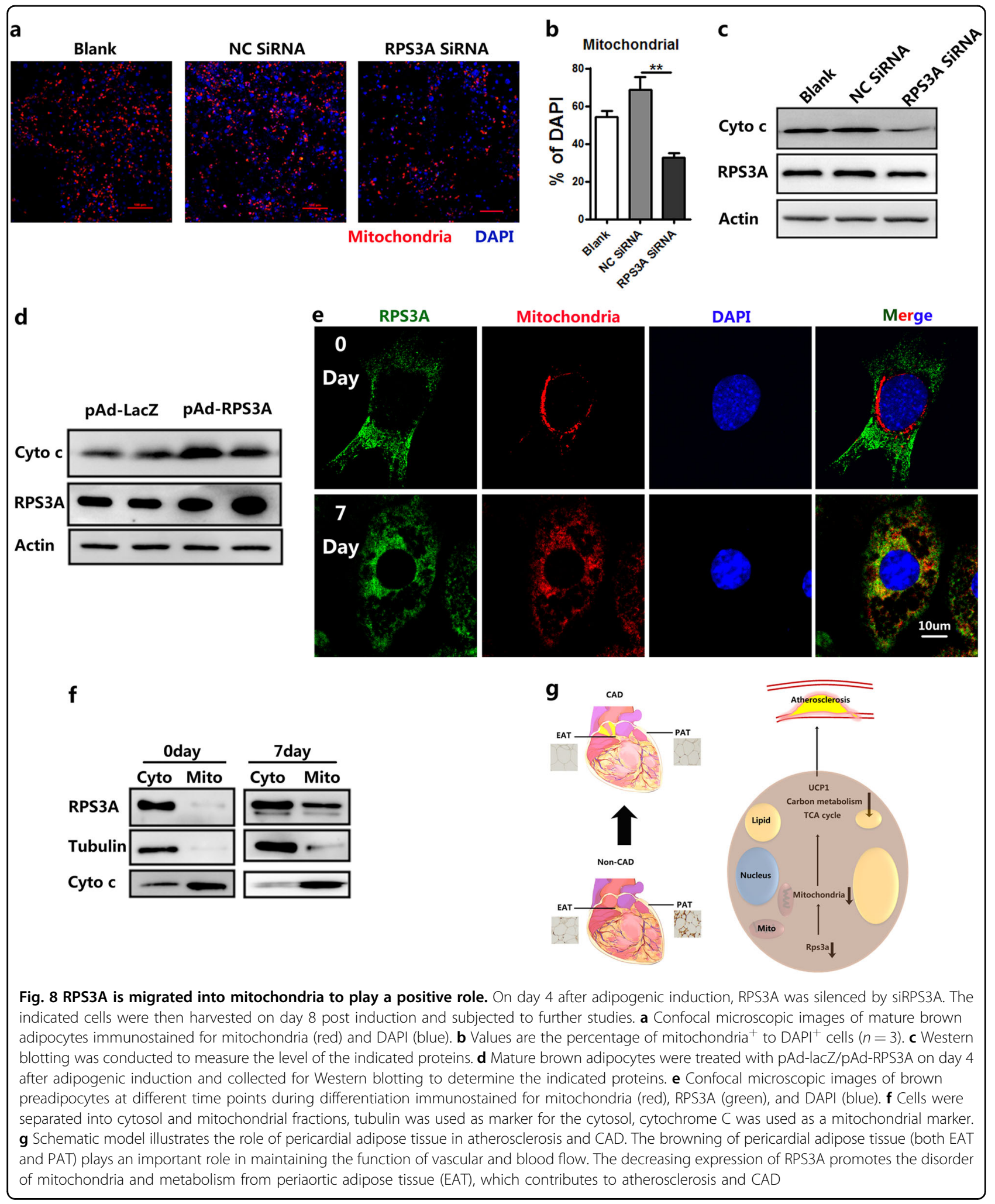

males (Fig. 2c, d). As a result, there is more browning of pericardial adipose tissue in non-CAD patients, especially in the PAT from female.
The underlying pathological process of CAD is atherosclerosis, which was previously considered a cholesterol deposition disease, and was associated with hypertension, 
smoking, diabetes, and obesity ${ }^{39,40}$. However, our findings demonstrated that only age, sex, waist size, and hypertension were strongly associated with $\mathrm{CAD}$, in the context of patients older than 55, which prompted us to explore other mechanisms of CAD. In the last decade, BAT has been considered a promising therapeutic target for obesity and associated metabolic disorders ${ }^{41,42}$. The anti-obesity potential of BAT has been irrefutably proven in murine studies and shown in human studies ${ }^{43,44}$. Moreover, BAT activation is beneficial and not deleterious for plasma cholesterol metabolism and atherosclerosis development ${ }^{9}$. However, the effect of BAT activation in human pericardial adipose on atherosclerosis and its relationship to CAD remains controversial to date. Here, we present evidence that BAT activation in pericardial adipose tissue was decreased in CAD patients. Using the SYNTAX score method, we identified that patients with the highest SYNTAX score had lower expression of UCP1, which highlights strong association of UCP1 expression in pericardial adipose tissue with vascular function. In addition, many retrospective analyses have shown that men account for a significantly greater proportion of CAD cases compared with women ${ }^{45}$. This is consistent with our study, in which the ratio of CAD was $58.8 \%$ among males and only $33.3 \%$ among females. The potential mechanism may be explained by BAT activation in females.

Mitochondria are cytoplasmic organelles in human and animal cells where many distinct metabolic processes take place, including TCA cycle, pyruvate decarboxylation, oxidative decarboxylation of fatty acids ( $\beta$-oxidation), and degradation of branched amino acids ${ }^{46}$. In addition, specific functions performed by brown fat convert mitochondrial energy into heat in adaptive thermogenesis ${ }^{47}$. Many rodents and human studies have found that obesity and insulin resistance is strongly associated with decreased mitochondria contents in brown or beige adipocytes $^{48-50}$. Moreover, other studies also found a decrease in TCA cycle and $\beta$-oxidation gene signatures and an increase in inflammation signatures in the insulinresistant state, while these gene expression changes were reversed after TZD treatment ${ }^{51,52}$. Here, our findings demonstrated that genes related to the citric acid cycle were under-expressed in the pericardial adipose tissue from CAD patients. Our findings are consistent with a published report showing that abnormal glucose metabolism is a core aspect of metabolic syndrome associated with high risk for heart disease ${ }^{21}$.

EAT is the fat derived from aortic root, while PAT is the fat surrounding the parietal pericardium. In the clinic, the presence of aortic atherosclerosis can be used as an additional marker for predicting CAD. Based on these facts, we analyze the proteins that were changed specifically in EAT. From our proteomic data, five proteins, Col1a1, RPS3A, HSD17B12, Crip1, and LCN2, were changed specially in EAT (Supplementary Fig. S3d and e). We used real-time PCR to validate the five candidates in EAT from non-CAD and CAD patients. RPS3A is obviously decreased in the CAD patients. Given the multifunction of ribosome protein, we chose RPS3A to explore its functional role during brown adipocyte differentiation. RPS3A is a component of the $40 \mathrm{~S}$ ribosome small subunit and plays a critical role in cell transformation, growth, and many cancers ${ }^{53}$. Over the past decade, more than a dozen ribosomal proteins have been shown to be involved in various physiological and pathological processes $^{54}$. RPS3 is a ribosome protein in the mitochondria, but not as a component of the mitochondrial $28 \mathrm{~S}$ subunit. When mitochondrial damages accumulate, RPS3 accumulates in the mitochondria through direct interaction with the cytoplasmic domain of Tom70 to repair damaged $\mathrm{DNA}^{55}$. Indeed, we found that knockdown of RPS3A in brown adipocytes decreased the number of mitochondria, as well as the function of mitochondria. The decreased browning of epicardial adipocyte may be due to the decreased expression of RPS3A in EAT, while the decreased browning of paracardial adipocytes may be the secondary phenomenon. Brown fat exerts effects on energy metabolism beyond its role in fuel oxidation. Also, it can serve as a source of endocrine factors $^{56}$. Using co-culture of PVAT-derived CM and endothelial cells, we found the anti-inflammation effect of PVAT on endothelial cells, especially the mature brown adipocytes overexpressing RPS3A. In addition, injection of adenovirus expressing RPS3A shRNA into periaortic adipose tissue damaged the vascular structure and induced inflammation.

Most cohort studies have shown that high blood pressure and increased serum TC increase the risk of developing CAD in men and women in a graded fashion. Besides these factors, there were still many people suffering from CAD without these factors. The results of our study highlight a subpopulation of individuals who display a metabolism disorder signature in their pericardial adipose tissue, which might contribute to their state of coronary artery disease. It is possible that individuals with less brown adipocytes are more at risk for the development of atherosclerosis and CAD. Understanding the mechanism that leads to these differences will equip us with important targets to help stem the tide of such a debilitating disease.

\section{Materials and methods \\ Patients}

Fifty-eight patients had angiographic evidence of critical coronary atherosclerosis (CAD) with stenosis greater than $50 \%$ in the left main artery or more than $75 \%$ in other arteries, involving one, two, or three vessels requiring elective coronary artery bypass grafting (CABG). The 
second group of 64 control patients without CAD had chronic valvular heart disease with or without stenosis less than $50 \%$ in any vessel requiring valve replacement but not CABG. The decision to perform heart surgery was made by the attending cardiologist and cardiac surgeon.

\section{Fat samples}

After the thorax had been opened and before heparinization and cardiopulmonary bypass, fat $(0.2 \mathrm{~g}$ to $1 \mathrm{~g})$ was obtained from the following sites: EAT over the aortic root; PAT from the inner midcourse and the diaphragm; and thoracic sc adipose tissue (Sub) from the sternotomy incision at the manubrium sterni. Fat samples were trimmed of connective tissue and superficial blood vessels, bisected, and stored separately at $-80^{\circ} \mathrm{C}$.

\section{SYNTAX score}

The SYNTAX score II was designed for risk assessment of patients with complex CAD for the purpose of choosing the optimal revascularization method (surgical or percutaneous). It combines the extension and complexity of native CAD with six clinical variables (gender, age, left ventricular ejection fraction, peripheral vascular disease, creatinine clearance, and chronic obstructive pulmonary disease) and was found to be a predictor of 4year mortality ${ }^{57}$.

\section{Mice}

Male apolipoprotein $\mathrm{E}$ deficient $\left(\mathrm{ApoE}^{-/-}\right)$mice were purchased from the Model Animal Research Center of Nanjing University (Nanjing, Jiangsu, China). Mice were maintained on a normal chow diet (ND) or HFD for 4 months to induce atherosclerosis. For RPS3A knockdown in periaortic adipose tissue assay, 8-week-old male $\mathrm{ApoE}^{-1-}$ mice were maintained on a HFD for 1 week before tranverse aortic constriction was performed ${ }^{58}$. Briefly, animals were anesthetized with $2 \%$ isoflurane inhalation, intubated, and ventilated with $100 \%$ oxygen $(0.7 \mathrm{~mL} / 100 \mathrm{~g}$ bodyweight, $90 / \mathrm{min})$, and placed in a supine position. A $2-\mathrm{cm}$ median cut along the neck was made and the underlying sternum was revealed. Then a $1.5-\mathrm{cm}$ median hemi-sternotomy was performed, and the prominent thymus was carefully put away to preserve the underlying structures. After isolating the aorta from the pulmonary trunk, a $10-\mu \mathrm{L}$ Hamilton syringe was placed around the aortic arch to inject adenovirus. Animal handling and experimental procedures were performed following approval from the Institute of Health Sciences Institutional Animal Care and Use Committee.

\section{Protein and peptide sample preparation}

Tissues from CAD and non-CAD patients were lysed by mixing with $150 \mu \mathrm{L}$ solution containing $4 \%$ sodium dodecyl sulfate (SDS), $100 \mathrm{mMTris} / \mathrm{HCl} \mathrm{pH}$ 7.6, $0.1 \mathrm{M}$
DTT (SDT lysis solution) followed by incubation at $95^{\circ} \mathrm{C}$ for $3 \mathrm{~min}$. The DNA was sheared by sonication to reduce the viscosity of the sample. Before sample processing, the lysate was clarified by centrifugation at $16,000 \times g$ for 5 min. The protein content was determined by measuring tryptophan fluorescence using the Cary Eclipse Fluorescence Spectrometer (Varian, Palo Alto, USA) as previously reported ${ }^{59}$.

The protein samples were digested by sequencing grade modified trypsin (1:50) using the Filter-Aided Sample Preparation protocol ${ }^{60}$. Peptide samples were cleaned of salts and residual oil using STop and Go Extraction tips, dried, and then stored at $-80^{\circ} \mathrm{C}$ before analysis.

\section{LC-MS/MS analysis}

Each peptide sample was analyzed on the nano HPLCLTQ-Orbitrap-Veloshybrid mass spectrometer (Thermo Electron Finnigan, San Jose, CA, USA). Peptide mixtures were separated through a nano-emitter column $(15 \mathrm{~cm}$ length, $75 \mu \mathrm{M}$ inner diameter) packed in-house with $3 \mu \mathrm{M}$ C18 ReproSil particles (Dr. Maisch GmbH) and introduced into the mass spectrometer using a nanoelectrospray ion source (source voltage, 1.7-2.2 kV). A linear gradient from 4 to $30 \%$ buffer B (buffer A, $0.1 \%$ formic acid in ddH2O; buffer B, $0.1 \%$ formic acid in acetonitrile) over $120 \mathrm{~min}$ was used for peptide separation at a flow rate of $250 \mathrm{~nL} / \mathrm{min}$. Collision-induced dissociation (CID) mode was performed on the LTQ-OrbitrapVelosmass spectrometer (Thermo Fisher Scientific, Waltham, MA, USA), and a full scan was acquired at a target value of $1,000,000$ ions with resolution $R=60,000$ at $\mathrm{m} / z 400$. The top 20 ions were selected at an isolation window of $2.0 \mathrm{~m} /$ $z$ units and accumulated to an AGC target value of $3 \mathrm{e} 4$ for tandem mass spectrometry (MS/MS) sequencing. Dynamic exclusion was enabled to void choosing former target ions for $120 \mathrm{~s}$, and lock-mass was enabled using 445.120025. Raw MS data were processed with MaxQuant software version 1.5.2.8 using the default settings with minor changes: oxidation (methionine) and acetylation (protein $\mathrm{N}$-term) were selected as variable modifications, and carbamidomethyl (C) was selected as fixed modification. Database searching was performed using the Andromeda search engine against Swiss-Prot Homo sapiens sequence database (downloaded Nov 2015, 20,196 protein entries), concatenated with known contaminants and reversed sequences of all entries ${ }^{61}$. A false discovery rate of less than 0.01 for proteins and peptides, and a minimum peptide length of 7 amino acids were required.

\section{Functional analysis}

To explore the potential EAT or PAT-associated biological functions between $\mathrm{CAD}$ and non-CAD cases, we mapped the differentially expressed proteins into known molecular sets with Kyoto Encyclopedia of Genes and 
Genomes (KEGG) pathway or Gene Ontology Cellular Component (GO-CC) term annotations. We estimated enrichment significance of specific proteins in each KEGG or GO-CC term based on the hypergeometric test ${ }^{62}$. Therefore, significantly enriched terms were chosen by a $p$ value less than 0.05 .

\section{IHC and IF}

Adipose tissue obtained was fixed in $4 \%$ paraformaldehyde overnight, embedded in paraffin, and cut into 4-mmthick sections. IHC was performed according to the Vecta Stain Elite ABC kit protocols (Vector Laboratories, Burlingame, CA, USA). Antibody staining was visualized with the enhanced DAB kit (Vector Laboratories). The brown reaction product was quantified using Image-Pro Plus software. For IF staining, mature adipocytes were fixed in $4 \%$ paraformaldehyde for $15 \mathrm{~min}$, pre-incubated in blocking buffer (10\% normal donkey serum in phosphatebuffered saline (PBS)) for $30 \mathrm{~min}$ at room temperature, and incubated sequentially with primary and secondary antibodies diluted in blocking buffer. After washing with PBS, the samples were counterstained with DAPI. Cells were placed on slides using cover slips and mounting medium (SouthernBiotech, Burlingame, CA, USA), and examined under a fluorescence microscope (Nikon, Tokyo, Japan).

\section{mRNA isolation and assays}

Total RNA were extracted by Trizol (Life Technologies, Carlsbad, CA, USA) and stored in DEPC $\mathrm{H}_{2} \mathrm{O}$ at $-20^{\circ} \mathrm{C}$. cDNA was synthesized from total RNA with RevertAid First Strand cDNA Synthesis Kit (Thermo Scientific). Real-time PCR was performed using Power SYBR Green qPCR Master Mix (Life Technologies) in 7500 Real Time PCR system (Applied Biosystems, San Francisco, CA, USA). The relative abundance of mRNAs was calculated with $18 \mathrm{~S}$ mRNA as the invariant control. The primers were from PrimerBank (http://pga.mgh.harvard.edu/ primerbank/). Primers are listed in Supplementary Table S2.

\section{Brown preadipocytes maintenance, differentiation, and treatment}

Immortalized brown preadipocytes were generated previously $^{63}$. Briefly, brown preadipocytes were maintained in DMEM medium supplemented with 10\% FBS (Gibco, Gaithersburg, MD, USA) and pen/strep, and were cultured in a $5 \% \mathrm{CO}_{2}$ atmosphere. After seeding onto plates for $24 \mathrm{~h}$, induction cocktail $(20 \mathrm{nM}$ insulin and $1 \mathrm{nM} \mathrm{T3}$ ) was added to the cell culture medium and incubated for 2 days. Then (designated as day 0 ), differentiation cocktail $(20 \mathrm{nM}$ insulin, $0.5 \mu \mathrm{M}$ dexamethasone, and $0.5 \mathrm{mM}$ 3-isobutyl-1-methyl-xanthine, $125 \mu \mathrm{M}$ indometacin and $1 \mathrm{nM}$ T3 for $48 \mathrm{~h}$ ) was added to the cells in fresh media. After $48 \mathrm{~h}$, the media was changed to DMEM supplemented with $10 \%$ FBS containing $20 \mathrm{nM}$ insulin and $1 \mathrm{nM}$ T3. The medium was refreshed every other day. Eight days after induction (designated as Day 8), cells was harvested for following analyses.

\section{Oxygen consumption assays}

Oxygen consumption rate (OCR) in EAT, PAT, and SAT was measured using the Seahorse XFe Extracellular Flux Analyzer (Agilent) in a 24-well plate. Adipose tissues $(2 \mathrm{mg}$ for EAT and PAT, $4 \mathrm{mg}$ for SAT) were placed into XF24 Islet Capture Microplates. For cultured adipocytes, OCR was measured in $1 \times 10^{4}$ differentiated adipocytes that were treated with pAd-LacZ shRNA, pAdRPS3A shRNA, pAd-LacZ, and pAd-RPS3A for $24 \mathrm{~h}$. Cells were subjected to the mitochondrial stress test by adding oligomycin $(10 \mu \mathrm{M}$ for tissue and $1 \mu \mathrm{M}$ for cells) followed by carbonyl cyanide4-(trifluoromethoxy) phenylhydrazone (FCCP, $9 \mu \mathrm{M}$ for tissue and $1 \mu \mathrm{M}$ for cells) and antimycin/rotenone $(12 \mu \mathrm{M}$ for tissue and $1 \mu \mathrm{M}$ for cells).

\section{Western blot analysis}

Cultured cells or tissues were prepared with the T-PER tissue protein extraction reagent (2\% SDS and $60 \mathrm{mM}$ Tris $\mathrm{HCl}, \mathrm{pH}$ 6.8) with the cocktail of proteinase inhibitors (Roche, Indianapolis, IN, USA) in it. The total protein tissue or cultured cells were homogenized in lysis buffer and were loaded onto the gel $(20-40 \mu \mathrm{g})$ for electrophoresis. Proteins then were transferred onto $10 \%$ SDS-PAGE (sodium dodecylsulfate polyacrylamide gel electrophoresis) and were then transferred to PVDF membranes (Bio-Rad, Hercules, CA, USA). The electroblotted membranes were blocked by TBS containing 5\% non-fat milk (Santa Cruz, Dallas, TX, USA) and were probed with primary antibodies overnight at $4{ }^{\circ} \mathrm{C}$ and immunoblotted with specific antibodies. Primary antibodies against the following proteins were used: UCP1, PGC1 $\alpha$, PRDM16, Cidea (Abcam, Cambridge, UK), PPARY (CST, Danvers, MA, USA), 422/Ap2 (Santa Cruz Biotechnology), RPS3A (ProteinTech Group, Rosemont, IL, USA), Cyto c (CST), Actin (Sigma, St. Louis, USA).

\section{Adipocyte-derived CM-dependent vascular inflammation}

The mature brown adipocytes were pretreated with adenovirus expressing LacZ or RPS3A for $6 \mathrm{~h}$, after which the medium was replaced. After $12 \mathrm{~h}$, the adipocytederived conditioned medium (ACM) was collected. The descending thoracic aorta of mice without PVAT was cut into 2-mm ring segments and suspended in CM for culture, and the medium was replaced every day. After 2 days of culture, the vascular cells were collected for RNA extraction. 


\section{RNA interference}

Synthetic siRNA oligonucleotides specific for RPS3A and Crip1 mRNA were designed and synthesized by invitrogen Stealth ${ }^{\mathrm{TM}}$ RNAi. The sequence was as follow:

RPS3A Stealth ${ }^{\mathrm{TM}}$ RNAi: CCGGAAGAAGATGATGGA AATTT.

Crip1 Stealth ${ }^{\mathrm{TM}}$ RNAi: CCCTGCCTGAAGTGCGAG AAATT.

\section{Adenoviral expression vectors and infection}

The adenoviral expression vector $\mathrm{pAd} / \mathrm{CMV} / \mathrm{V} 5-\mathrm{DEST}$ (Invitrogen, Carlsbad, CA, USA) encoding the RPS3A gene was constructed according to the manufacturer's protocol. The RPS3A sequence was amplified using the primers 5'-CACCATGGCGGTCGGCAAG-3' (forward) and 5'-TTACACTGATTCTTGGA-3' (reverse).

\section{Statistical analyses}

All results are presented as the mean \pm SEM. A nonpaired Student's $t$ test was used for these analyses. A difference was considered significant at ${ }^{*} p<0.05$, $* * 0<0.01$, and ${ }^{* * * *} p<0.001$.

\section{Acknowledgements}

We thank J. Wu and Y.Z. Zou from Shanghai Institute of Cardiovascular Diseases, Zhongshan Hospital, Fudan University (Shanghai, China), for assistance with cardiac procedure like TAC in animal models. We appreciate Ms. Y. Qin with Art and Design College, Shenyang Ligong University (Liaoning, China), for her assistance with drawing the Schematic model in Fig. 8. This study was supported by the National Natural Science Foundation of China (grant no. 31701254 to Y.T., grant nos. 81730021 and 31571471 to Q.-Q. T., grant no. 31670787 to S.-W.Q., and grant no. 91539124 to R.Z.) and China Postdoctoral Science Foundation (grant no. 1322094 to Y.T.).

\section{Author details}

'Key Laboratory of Metabolism and Molecular Medicine of Chinese Ministry of Education, Department of Biochemistry and Molecular Biology of School of Basic Medical Sciences and Department of Endocrinology of Zhongshan Hospital, Fudan University, 200032 Shanghai, China. ${ }^{2}$ Department of Cardiothoracic Surgery, Xinhua Hospital, Shanghai Jiaotong University of Medicine College, 200032 Shanghai, China. ${ }^{3}$ Key Laboratory of Systems Biology, Institute of Biochemistry and Cell Biology, Shanghai Institutes for Biological Sciences, Chinese Academy of Sciences, Shanghai, China

\section{Authors' contributions}

Y.T., Y.H., and Q.Q.T. designed the research; Y.T., Y.H., C.L., W.J.M., Y.Z., C.H.L., F.C. Z., J.B.P., and Y.N.W. performed the research; Y.T., S.W.Q., H.Y.H., J.M., R.Z., and Q. Q.T. analyzed the data; Y.T., C.L., and Q.Q.T. wrote the paper.

\section{Conflict of interest}

The authors declare that they have no conflict of interest.

\section{Publisher's note}

Springer Nature remains neutral with regard to jurisdictional claims in published maps and institutional affiliations.

Supplementary Information accompanies the paper at (https://doi.org/ 10.1038/s41421-018-0041-2).

Received: 8 November 2017 Revised: 27 May 2018 Accepted: 29 May 2018 Published online: 21 August 2018

\section{References}

1. Van Gaal, L. F., Mertens, I. L. \& De Block, C. E. Mechanisms linking obesity with cardiovascular disease. Nature 444, 875-880 (2006).

2. Fox, C. S. et al. Abdominal visceral and subcutaneous adipose tissue compartments: association with metabolic risk factors in the Framingham Heart Study. Circulation 116, 39-48 (2007).

3. Frühbeck, G. et al. BAT: a new target for human obesity? Trends Pharmacol. Sci. 30, 387-396 (2009).

4. Nedergaard, J., Bengtsson, T. \& Cannon, B. Unexpected evidence for active brown adipose tissue in adult humans. Am. J. Physiol. Endocrinol. Metab. 293 E444-E452 (2007).

5. Cypess, A. M. et al. Identification and importance of brown adi入ose tissue in adult humans. N. Engl. J. Med. 360, 1509-1517 (2009).

6. Virtanen, K. A. et al. Functional brown adipose tissue in healthy adults. N. Engl. J. Med. 360, 1518-1525 (2009).

7. Chang, L. et al. Loss of perivascular adipose tissue on peroxisome proliferatoractivated receptor-gamma deletion in smooth muscle cells impairs intravascular thermoregulation and enhances atherosclerosis. Circulation $\mathbf{1 2 6}$ 1067-1078 (2012)

8. Szasz, T. \& Webb, R. C. Perivascular adipose tissue: more than just structural support. Clin. Sci. (Lond.) 122, 1-12 (2012).

9. Berbee, J. F. et al. Brown fat activation reduces hypercholesterolaemia and protects from atherosclerosis development. Nat. Commun. 6, 6356 (2015).

10. Mazurek, T. \& Opolski, G. Pericoronary adipose tissue: a novel therapeutic target in obesity-related coronary atherosclerosis. J. Am. Coll. Nutr. 34, 244-254 (2015).

11. Cheung, L. et al. Human mediastinal adipose tissue displays certain characteristics of brown fat. Nutr. Diabetes 3, e66 (2013).

12. Sacks, H. S. et al. Adult epicardial fat exhibits beige features. J. Clin. Endocrinol. Metab. 98, E1448-E1455 (2013).

13. Sacks, H. S. et al. Uncoupling protein-1 and related messenger ribonucleic acids in human epicardial and other adipose tissues: epicardial fat functioning as brown fat. J. Clin. Endocrinol. Metab. 94, 3611-3615 (2009).

14. Harada, K. et al. Impact of abdominal and epicardial fat on the association between plasma adipocytokine levels and coronary atherosclerosis in nonobese patients. Atherosclerosis 237, 671-676 (2014).

15. Ong, K. L. et al. Relationship of pericardial fat with biomarkers of inflammation and hemostasis, and cardiovascular disease: the Multi-Ethnic Study of Atherosclerosis. Atherosclerosis 239, 386-392 (2015).

16. Kocaman, S. A. et al. An increase in epicardial adipose tissue is strongly associated with carotid-intima media thickness and atherosclerotic plaque, but LDL only with the plaque. Anatol. J. Cardiol. 17, 56-63 (2017).

17. Lutsch, G. et al. Immunoelectron microscopic studies on the location of ribosomal proteins on the surface of the 405 ribosomal subunit from rat liver. Eur. J. Cell Biol. 51, 140-150 (1990).

18. Kho, C. J. \& Zarbl, H. Fte-1, a v-fos transformation effector gene, encodes the mammalian homologue of a yeast gene involved in protein import into mitochondria. Proc. Natl Acad. Sci. USA 89, 2200-2204 (1992).

19. Shuda, M. et al. Enhanced expression of translation factor mRNAs in hepatocellular carcinoma. Anticancer Res. 20, 2489-2494 (2000).

20. Lim, K. H. et al. RPS3a over-expressed in HBV-associated hepatocellular carcinoma enhances the HBx-induced NF-kappaB signaling via its novel chaperoning function. PLOS ONE 6, e22258 (2011).

21. Selvin, E., M.S.B.G. Meta-analysis glycosylated hemoglobin and cardiovascular disease in diabetes mellitus. Ann. Intern. Med. 141, 421-431 (2004).

22. Kizilay, M. O. et al. Mitochondrial oxidative stress reduces the immunopotency of mesenchymal stromal cells in adults with coronary artery disease. Circ. Res. 122, 255-266 (2018).

23. Mackenzie, R. M. et al. Mitochondrial reactive oxygen species enhance AMP-activated protein kinase activation in the endothelium of patients with coronary artery disease and diabetes. Clin. Sci. (Lond), 124, 403-411 (2013).

24. Yüce, G., Türkvatan, A. \& Yener, Ö. Can aortic atherosclerosis or epicardial adipose tissue volume be used as a marker for predicting coronary artery disease? J. Cardiol. 65, 143-149 (2015).

25. Tang, Q. Q. et al. Sequential phosphorylation of CCAAT enhancer-binding protein by MAPK and glycogen synthase kinase 3 is required for adipogenesis. Proc. Natl Acad. Sci. USA 102, 9766-9771 (2005).

26. Sarjeant, K. \& Stephens, J. M. Adipogenesis. Cold Spring Harb. Perspect. Biol. 4, a008417-a008417 (2012) 
27. Guo, L., Li, X. \& Tang, Q. Transcriptional regulation of adipocyte differentiation: a central role for CCAAT/enhancer-binding protein (C/EBP) $\beta$. J. Biol. Chem. 290, 755-761 (2015).

28. Zhang, Y. Y. et al. Transcriptional activation of histone $\mathrm{H} 4$ by $\mathrm{C} / \mathrm{EBPb}$ beta during the mitotic clonal expansion of 3T3-L1 adipocyte differentiation. Mol. Biol. Cell 22, 2165-2174 (2011)

29. Rosen, E. D. et al. PPARY is required for the differentiation of adipose tissue in vivo and in vitro. Mol. Cell 4, 611-617 (1999).

30. Behrens, G. M. et al. Clinical impact of HIV-related lipodystrophy and metabolic abnormalities on cardiovascular disease. AIDS 17(Suppl. 1), S149-S154 (2003)

31. Wang, G., Zhao, X. \& Lin, J. D. The brown fat secretome: metabolic functions beyond thermogenesis. Trends Endocrinol. Metab. 26, 231-237 (2015).

32. Lin, Z. et al. Fibroblast growth factor 21 prevents atherosclerosis by suppression of hepatic sterol regulatory element-binding protein- 2 and induction of adiponectin in mice. Circulation 131, 1861-1871 (2015).

33. Dawkins, M. J. \& Scopes, J. W. Non-shivering thermogenesis and brown adipose tissue in the human new-born infant. Nature 206, 201-202 (1965).

34. Himms-Hagen, J. Thermogenesis in brown adipose tissue as an energy buffer. Implications for obesity. N. Engl. J. Med. 311, 1549-1558 (1984).

35. Bartelt, A. et al. Brown adipose tissue activity controls triglyceride clearance Nat. Med. 17, 200-205 (2011).

36. van Dam, A. D. et al. Targeting white, brown and perivascular adipose tissue in atherosclerosis development. Eur. J. Pharmacol. 816, 82-92 (2017).

37. De Lorenzo, F. et al. Central cooling effects in patients with hypercholesterolaemia. Clin. Sci. (Lond.) 95, 213-217 (1998).

38. Rodriguez, A. M. et al. Sex-dependent dietary obesity, induction of UCPs, and leptin expression in rat adipose tissues. Obes. Res. 9, 579-588 (2001).

39. Moore, K. J., Sheedy, F. J. \& Fisher, E. A. Macrophages in atherosclerosis: a dynamic balance. Nat. Rev. Immunol. 13, 709-721 (2013).

40. Witztum, J. L. \& Lichtman, A. H. The influence of innate and adaptive immune responses on atherosclerosis. Annu. Rev. Pathol. 9, 73-102 (2014).

41. Hany, T. F. et al. Brown adipose tissue: a factor to consider in symmetrical tracer uptake in the neck and upper chest region. Eur. J. Nucl. Med. Mol. Imaging 29, 1393-1398 (2002).

42. Zingaretti, M. C. et al. The presence of UCP1 demonstrates that metabolically active adipose tissue in the neck of adult humans truly represents brown adipose tissue. FASEB J. 23, 3113-3120 (2009).

43. Harms, M. \& Seale, P. Brown and beige fat: development, function and therapeutic potential. Nat. Med. 19, 1252-1263 (2013).

44. Yoneshiro, T. et al. Recruited brown adipose tissue as an antiobesity agent in humans. J. Clin. Invest. 123, 3404-3408 (2013).

45. Ding, Z . et al. Retrospective analysis of 769 cases of sudden cardiac death from 2006 to 2015: a forensic experience in China. Forensic Sci. Med. Pathol. 13, 336-341 (2017)

46. Wang, C. H., Wang, C. C. \& Wei, Y. H. Mitochondrial dysfunction in insulin insensitivity: implication of mitochondrial role in type 2 diabetes. Ann. N. Y. Acad. Sci. 1201, 157-165 (2010).
47. Cedikova, M. et al. Mitochondria in white, brown, and beige adipocytes. Stem Cells Int.2016,6067349 (2016)

48. Qian, S. W. et al. BMP4-mediated brown fat-like changes in white adipose tissue alter glucose and energy homeostasis. Proc. Natl Acad. Sci. USA 110 E798-E807 (2013).

49. Kissig, M. et al. PRDM16 represses the type I interferon response in adipocytes to promote mitochondrial and thermogenic programing. EMBO J. $\mathbf{3 6}$, 1528-1542 (2017).

50. Porter, C. Quantification of UCP1 function in human brown adipose tissue. Adipocyte. 6, 1-8 (2017).

51. Qatanani, M. et al. Inverse regulation of inflammation and mitochondrial function in adipose tissue defines extreme insulin sensitivity in morbidly obese patients. Diabetes 62, 855-863 (2013).

52. Sears, D. D. et al. Mechanisms of human insulin resistance and thiazolidinedione-mediated insulin sensitization. Proc. Natl Acad. Sci. USA 106 18745-18750 (2009)

53. Zhou, X. et al. Ribosomal proteins: functions beyond the ribosome. J. Mol. Cell Biol. 7, 92-104 (2015).

54. Wang, W. et al. Ribosomal proteins and human diseases: pathogenesis, molecular mechanisms, and therapeutic implications. Med. Res. Rev. 35 225-285 (2015).

55. Kim, Y., Kim, H. D. \& Kim, J. Cytoplasmic ribosomal protein S3 (rpS3) plays a pivotal role in mitochondrial DNA damage surveillance. Biochim. Biophys. Acta 1833, 2943-2952 (2013).

56. Wang, G. X. et al. The brown fat-enriched secreted factor Nrg4 preserves metabolic homeostasis through attenuation of hepatic lipogenesis. Nat. Med. 20, 1436-1443 (2014).

57. Farooq, V. et al. Anatomical and clinical characteristics to guide decision making between coronary artery bypass surgery and percutaneous coronary intervention for individual patients: development and validation of SYNTAX score II. Lancet 381, 639-650 (2013).

58. Schaefer, A. et al. A new animal model for investigation of mechanical unloading in hypertrophic and failing hearts: combination of transverse aortic constriction and heterotopic heart transplantation. PLOS ONE 11, e0148259 (2016).

59. Nielsen, P. A. et al. Proteomic mapping of brain plasma membrane proteins. Mol. Cell. Proteomics 4, 402-408 (2005).

60. JR, W. N. et al. Universal sample preparation method for proteome analysis. Nat. Methods 6, 359-362 (2009).

61. Cox, J. et al. Andromeda: a peptide search engine integrated into the MaxQuant environment. J. Proteome Res. 10, 1794-1805 (2011).

62. Huang, D. W., Sherman, B. T. \& Lempicki, R. A. Bioinformatics enrichment tools: paths toward the comprehensive functional analysis of large gene lists. Nucleic Acids Res. 37, 1-13 (2009).

63. Pan, D. et al. Jmjd3-mediated H3K27me3 dynamics orchestrate brown fat development and regulate white fat plasticity. Dev. Cell 35, 568-583 (2015). 